\title{
A NOTE ON A CONJECTURE OF DUVAL AND STURMIAN WORDS
}

\author{
Filippo Mignosi ${ }^{1}$ And Luca Q. Zamboni ${ }^{2}$
}

\begin{abstract}
We prove a long standing conjecture of Duval in the special case of Sturmian words.
\end{abstract}

Mathematics Subject Classification. 68R15, 37B10.

Let $U$ be a nonempty word on a finite alphabet $\mathcal{A}$. A nonempty word $B$ different from $U$ is called a border of $U$ if $B$ is both a prefix and suffix of $U$. We say $U$ is bordered if $U$ admits a border, otherwise $U$ is said to be unbordered. For example, $U=011001011$ is bordered by the factor 011 , while 00010001001 is unbordered.

An integer $1 \leq k \leq n$ is a period of a word $U=U_{1} \ldots U_{n}$ if and only if for all $1 \leq i \leq n-k$ we have $U_{i}=U_{i+k}$. It is easy to see that $k$ is a period of $U$ if and only if the prefix $B$ of $U$ of length $n-k$ is a border of $U$ or is empty. Let $\lambda(U)$ denote the smallest period of $U$. Then $U$ is unbordered if and only if $\lambda(U)$ is equal to the length of $U$, that is $\lambda(U)=|U|=n$. We further denote by $\mu(U)$ the length of the longest unbordered factor of $U$. Clearly $U$ is unbordered if and only if $\mu(U)=|U|$.

In general, for any word $U$ we have $\lambda(U) \geq \mu(U)$ (cf. Prop. 2.2 of [3]). An interesting question is to ask for which words $U$ does equality hold. In [3] Duval shows that $\lambda(U)=\mu(U)$ whenever $|U| \geq 2 \lambda(U)-2$ (cf. Cor. 4.2 in [3]). These notions extend directly to infinite words. For an infinite word $\omega$, if $\mu(\omega)$ is finite, then $\omega$ is periodic of period $\mu(\omega)$. (cf. [2] and [4]). In [3] Duval conjectured that:

Conjecture 1 (Duval 1981). Let $U$ be an unbordered word on an alphabet $A$ and let $W$ be a word of length $2|U|$ beginning in $U$ and with the property that each factor of $W$ of length greater than $|U|$ is bordered. Then $W$ has period $|U|$, i.e., $W=U U$.

Keywords and phrases: Bordered words, Sturmian words.

1 Dipartimento di Matematica e Applicazioni, Università degli Studi di Palermo,

Via Archirafi 34, 90123 Palermo, Italia; e-mail: mignosi@dipmat.math.unipa.it

2 Department of Mathematics, P.O. Box 311430, University of North Texas, Denton,

TX 76203-1430, USA; e-mail: luca@unt.edu

(C) EDP Sciences 2002 
This is the latest and strongest in a series of conjectures reported in [2] and [4], that up to this day remain open.

In view of the above conjecture we say $W$ is a Duval extension of $U$ if $W$ begins in $U$ and each factor of $W$ of length greater than $|U|$ is bordered.

Remark. We do not know if the conjecture remains true under the weaker assumption that $|W|=2|U|-1$, in other words that if $U$ is unbordered, then each Duval extension $W$ of $U$ with $|W|=2|U|-1$ has period $|U|$. However there exists unbordered words $U$ which admit more than one Duval extension of length $2|U|-2$. For example, let $U$ be any word of the form $U=01 \mathrm{~V} 11$ where $V$ is any nonempty word on the alphabet $\{0,1\}$ with the property that $1 V 1$ contains no occurrences of 11 . Then clearly $U$ is unbordered. Moreover $01 \mathrm{~V} 1101 \mathrm{~V}$ and $01 \mathrm{~V} 11 \mathrm{~V} 10$ are both Duval extensions of $U=01 V 11$ and are distinct whenever $V$ is not of the form $(01)^{k} 0$ for $k \geq 0$.

In this note we verify Duval's conjecture in the special case that $U$ is an unbordered Sturmian word, that is when $U$ is an unbordered factor of an infinite Sturmian word on $\{0,1\}$. We actually prove something stronger:

Theorem 1. Let $U$ be an unbordered Sturmian word. Then every Duval extension of $U$ has period $|U|$.

Proof. Without loss of generality we can assume that $U$ begins in 0 and ends in 1 . We proceed by induction on $|U|$. The shortest unbordered word with this property is $U=01$ in which case the result of the theorem is easily verified. Next we suppose that $|U|>2$. If the symbol 0 occurs only once in $U$, then $U$ is necessarily of the form $01^{k}$ with $k \geq 1$, and in this case once again it is readily verified that any Duval extension of $U$ is a prefix of $01^{k} 01^{k} 01^{k} \ldots$ Thus we can assume that 0 occurs at least twice in $U$. Since $U$ is unbordered and Sturmian, $U$ is of the form $U=01^{k} V 01^{k+1}$ with $k \geq 0$ and $V$ a word (possibly empty). Let us suppose to the contrary that $U$ admits a Duval extension $\hat{U}$ of period different than $|U|$. Let $W$ denote the longest prefix of $\hat{U}$ with period equal to $|U|$. Then both $W 0$ and $W 1$ are Duval extensions of $U$, one of the two beeing a prefix of $\hat{U}$, the other beeing periodic of period $|U|$. Moreover we can write $W$ in the form $W=01^{k} V 01^{k+1} V^{\prime} 1^{k}=U V^{\prime} 1^{k}$ with $V^{\prime}$ a nonempty word beginning and ending in 0 .

Consider the morphism $\tau$ defined by $\tau(0)=01^{k}$ and $\tau(1)=01^{k+1}$. Let $Z$ be such that $W=Z 01^{k}$. Then $U$ is a prefix of $Z$ and both $U$ and $Z$ are concatenations of the words $\tau(0)$ and $\tau(1)$. Let $u$ and $z$ be such that $U=\tau(u)$ and $Z=\tau(z)$. Then $u$ is Sturmian (see Chap. 3 on Sturmian sequences in [5]) unbordered (since $U$ is unbordered), and $|u|<|U|$. Also, $u$ is a prefix of $z$ and the period of $z$ is equal to $|u|$ (since the period of $Z$ is equal to $|U|$ ).

We claim that both $z 0$ and $z 1$ are Duval extensions of $u$, which would yield a contradition since by induction hypothesis every Duval extension of $u$ has period $|u|$. To see that $z 1$ is a Duval extension of $u$ let $x$ be any suffix of $z 1$ of length greater than $|u|$. If $x$ begins in 1 then $x$ is bordered. If $x$ begins in 0 , set $X=\tau(x)$. Then $X$ is a suffix of $W 1$ beginning in $01^{k}$ and ending in $01^{k+1}$ and of length 
greater than $|U|$. Since $W 1$ is assumed to be a Duval extension of $U, X$ admits a border $B$. Moreover as $B$ begins in $01^{k}$ and ends in $01^{k+1}$ we can write $B=\tau(b)$ where $b$ is a border of $x$.

To see that $z 0$ is a Duval extension of $u$ we suppose that $x$ is a suffix of $z 0$ beginning in 1 and of length greater than $|u|$. Setting $X=\tau(x)$, we have that $X$ is a suffix of $W$ beginning in $01^{k+1}$ and of length greater than $|U|$. Writing $X=0 X^{\prime}$, we have that $X^{\prime} 0$ is a suffix of $W 0$. Since $\left|X^{\prime} 0\right|=|X|>|U|$ we have that $X^{\prime} 0$ admits a border $B$. It follows that $B$ begins in $1^{k+1}$ and ends in $01^{k} 0$. Thus $0 B$ is a suffix of $X^{\prime} 0$ and hence $0 B$ is a border of $0 X^{\prime} 0=X 0$. Setting $B=B^{\prime} 0$ we have that $0 B^{\prime}$ is a border of $X$ beginning in $01^{k+1} 0$ and ending in $01^{k}$. Moreover as $0 B^{\prime} 0$ is a prefix of $X$, it follows that $0 B^{\prime}=\tau\left(b^{\prime}\right)$ for some border $b^{\prime}$ of $x$.

This completes the proof of Theorem 1 .

A word $V$ is called a bispecial Sturmian word if $V$ is a palindrome and $0 V 1$ is Sturmian. Using an induction method similar to the one used in the proof of Theorem 1, one can show that a Sturmian word $U$ is unbordered if and only if $U$ is of the form $0 V 1$ or $1 V 0$ where $V$ is either empty or a bispecial Sturmian word. In [6] Pirillo obtained the following nice characterization of these words:

Theorem 2. Let $W$ be a (possibly) empty word on a finite alphabet $\mathcal{A}$, and let $a, b$ be distinct letters in $\mathcal{A}$. Then the words $a W b$ and $b W a$ are conjugate to one another if and only if $W$ is either empty or a bispecial Sturmian word on the alphabet $\{a, b\}$.

The method of proof of Theorem 1 may be generalized to a broader class of words which are also generated by substitution rules which "preserve borders". For instance the so-called Arnoux-Rauzy words of [1].

\section{REFERENCES}

[1] P. Arnoux and G. Rauzy, Représentation géométrique des suites de complexité $2 n+1$. Bull. Soc. Math. France 119 (1991) 199-215.

[2] R. Assous and M. Pouzet, Une Caractérisation des mots périodiques. Discrete Math. 25 (1979) $1-5$.

[3] J.P. Duval, Relationship between the Period of a Finite Word and the Length of its Unbordered Segments. Discrete Math. 40 (1982) 31-44.

[4] A. Ehrenfeucht and D.M. Silberger, Periodicity and Unbordered Segments of words. Discrete Math. 26 (1979) 101-109.

[5] Lothaire, Algebraic Combinatorics on Words, Chap. 9 Periodicity, Chap. 3 Sturmian Words. Cambridge University Press (to appear). Available at http://www-igm.univ-mlv.fr/berstel

[6] G. Pirillo, A rather curious characteristic property of standard Sturmian words, to appear in Algebraic Combinatorics, edited by G. Rota, D. Senato and H. Crapo. Springer-Verlag Italia, Milano (in press).

[7] F. Mignosi and P. Séébold, Morphismes sturmiens et règles de Rauzy. J. Théorie des Nombres de Bordeaux 5 (1993) 221-233.

[8] G. Rauzy, Mots infinis en arithmétique, in Automata on Infinite Words, edited by M. Nivat and D. Perrin. Lecture Notes in Comput. Sci. 192 (1985) 167-171.

[9] R. Risley and L.Q. Zamboni, A generalization of Sturmian sequences; combinatorial structure and transcendence. Acta Arith. 95 (2000).

Communicated by A. Restivo.

Received February 5, 2001. Accepted June, 2001. 